\section{The TGF- $\beta$ pathway: a pharmacological target in hepatocellular carcinoma?}

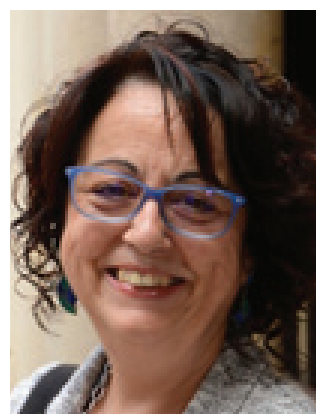

\author{
"...the TGF- $\beta$ pathway is a relevant druggable \\ target for patients with advanced \\ hepatocellular carcinoma."
}

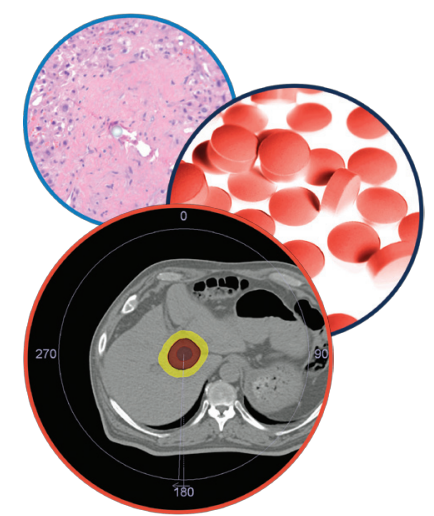

Hepatic Oncology

Isabel Fabregat ${ }^{*, 1} \&$ Gianluigi Giannelli²; on behalf of the IT-LIVER

\title{
Consortium
}

First draft submitted: 27 April 2017; Accepted for publication: 11 May 2017; Published online: 6 July 2017

The TGF- $\beta$ pathway plays essential roles in liver physiology and pathology. During liver development, TGF- $\beta$-driven signaling orchestrates liver-specific gene expression and differentiation of hepatoblasts to either hepatocytes or cholangiocytes. Early experiments in animal models already demonstrated that TGF- $\beta 1$ is required for the normal development and physiological functions of hepatocytes. In adulthood, strong evidence supports a crucial role for TGF- $\beta$ as cytostatic and apoptotic in hepatocytes, critical processes for the control of liver mass. However, despite these essential benefits, further investigations have yielded evidence that TGF- $\beta$ also plays major roles in chronic liver diseases, promoting fibrogenesis and influencing tumorigenesis at different levels [1].

TGF- $\beta$ is recognized as a profibrogenic cytokine, mainly due to its role in hepatic stellate cell activation and extracellular matrix production [2]. Recent results indicate that upregulation of the NADPH oxidase NOX4 would mediate TGF- $\beta$ effects [3]. The analysis of its role in liver tumorigenesis is more complex. Due to its cytostatic actions [1], TGF- $\beta$ could play a suppressor role in early stages of carcinogenesis. However, strong evidence suggests that later it activates antiapoptotic and proliferative signals, due to transactivation of tyrosine kinase receptors, such as PDGF [4] or EGF [5], allowing liver tumor cells to evade its antiproliferative and proapoptotic effects. Different conditions in the tumor microenvironment may also provide the signals for a switch in the role of TGF- $\beta$ in late hepatocarcinogenesis [6]. Once liver tumor cells overcome TGF- $\beta$ suppressor actions, they respond by undergoing the epithelial-mesenchymal transition (EMT) [7]. Mediated by an upregulation of specific transcription factors, EMT induces a downregulation of E-cadherin and other epithelial genes and upregulation of vimentin and other mesenchymal genes, as well as cytoskeleton remodeling, all of which contribute to enhance tumor cell migratory and invasive capacities [7].
KEYWORDS

- HCC • targeted therapy • TGF-beta 
“...TGF- $\beta$ also plays major roles in chronic liver diseases, promoting fibrogenesis and

influencing tumorigenesis at different levels."
Furthermore, TGF- $\beta$-induced plasticity moves liver tumor cells toward a higher migratory stemness phenotype [8].

While there is no doubt about the duality of TGF- $\beta$ actions, the predominant role of the TGF- $\beta$ pathway as promoter of hepatocarcinogenesis has been confirmed in different studies performed in animal models. Indeed, specific deletion of the TGF- $\beta$ receptor II (Tgfbr 2 ) in differentiated hepatocytes does not induce liver tumors, nor does it enhance the neoplastic response in a model of diethylnitrosamineinduced hepatocellular carcinoma (HCC) $[9,10]$, indicating that the suppressor function of TGF- $\beta$ is not so crucial to impair hepatocarcinogenesis. Contrary to previous expectations, deletion of $T g f b r 2$ in hepatocytes decreased the frequency of liver tumors in mice in the setting of $\mathrm{p} 53$ loss (Trp $53 \mathrm{KO} ; \operatorname{Tg} f b r 2 \mathrm{KO})$, indicating that TGF- $\beta$ promotes the formation of liver tumors that arise in the setting of $\mathrm{p} 53$ inactivation [9]. In humans, a classification was established, applying a comparative functional genomics approach according to the temporal TGF- $\beta$ gene expression signature established in mouse primary hepatocytes [11]. This novel approach successfully discriminated distinct subgroups of HCC, showing that a 'late' TGF- $\beta$ signature (expression of EMT, antiapoptotic and invasion related genes) correlated with an increased tumor recurrence and more invasive phenotype [11]. In this sense, attenuation of the TGF- $\beta$ pathway in vitro in human HCC cells does not inhibit proliferation or induce apoptosis, but strongly blocks their migration and invasion, as well as their stemness capacity $[8,12,13]$. Furthermore, functional studies have indicated that the WNT pathway activation, characteristic of a subpopulation of HCC tumors, is not only the consequence of $\beta$-catenin mutations, but may also be the result of TGF- $\beta$ activation [14]. In fact, patients with the worst survival show activation of the TGF- $\beta$ pathway that induces $\beta$-catenin nuclear translocation leading to WNT signaling, contributing to tumoral growth and progression. The phenotype of this subset of patients was also consistent with a late TGF- $\beta$ signature and features an EMT/ invasive phenotype [14].

All this considerable volume of preclinical and clinical evidence suggests that TGF- $\beta$ may be a promising pharmacological target in patients with HCC [15], reinforced by the fact that increased TGF- $\beta$ levels have been reported in HCC. In this sense, galunisertib (LY2157299), a selective ATP-mimetic inhibitor of TGF- $\beta$ receptor kinase I, blocks the canonical and noncanonical TGF- $\beta$ pathways. In the past, several different therapeutic strategies to block TGF- $\beta$ signaling have been investigated, including vaccines, monoclonal antibodies, antisense oligonucleotides and lastly small molecules inhibitors. One of the major difficulties has been to be able to rely on a drug that specifically inhibits the TGF- $\beta$ pathway, reducing the off target effects. From this point of view, galunisertib showed low cardiotoxicity in vivo but an efficient activity as a pSMAD2 inhibitor [16]. Based on these data, galunisertib has been further studied in a number of different experimental preclinical models, where it showed a high selective activity toward the canonical and noncanonical TGF- $\beta$ pathways [17], and is currently under investigation in different malignancies including pancreatic cancer and HCC $[18]$. The main therapeutic strategy for inhibiting TGF- $\beta$ in HCC depends on the fact that the underlying liver cirrhosis is in itself a premalignant disease, while the worsening liver fibrosis is a further negative prognostic factor. Therefore, inhibiting TGF- $\beta$ is at the same time a therapeutic strategy for reducing tumoral progression and also for decreasing the degree of liver fibrosis, restoring the liver homeostasis. For all these reasons, a Phase II clinical trial $\mathrm{H} 9 \mathrm{H}$ MC-JBAK (NCT01246986, http://clinicaltrials.gov) is currently ongoing, using galunisertib in patients with advanced HCC to test safety, time to progression and overall survival. The final results are awaited, but preliminary data already seem very promising. Unfortunately, in this trial a placebo-treatment arm has not been included, mainly because it was a Phase II study, but the robustness of the significance would have been improved. In the JBAK trial, HCC patients were enrolled without any stratification according with TGF- $\beta$-related biomarkers. Recently it has been reported that circulating TGF- $\beta$-related biomarkers are not associated with disease progression estimated by the Barcelona-Clinic Liver Cancer (BCLC) staging system, suggesting that the most suitable patients for treatment with galunisertib are not necessarily those with more advanced disease [19]. However, investigation of a large panel of serological and tissue biomarkers in patients enrolled in the JBAK trial is currently ongoing and the results will be available in the next few months. Nevertheless, in a preclinical model of HCC specimens cultured in the presence of TGF- $\beta$ and or galunisertib, new 
companion diagnostics have been investigated by next-Generation Sequencing-based Massive Analysis of cDNA Ends. Of the whole transcriptome only SKIL (SKI like protooncogene) and PMEPA1 (prostate transmembrane proein, androgen-induced 1) were strongly correlated with endogenous TGF- $\beta 1$ [20]. These data from preclinical experimental models are very important to move toward precision medicine and may serve as biomarkers for the stratification of HCC patients undergoing treatment with galunisertib [20].

Another crucial point for targeting the TGF- $\beta$ is its modulatory effect on the immune system within the microenvironment [6]. It is well known that TGF- $\beta$ displays an immunosuppressive effect, allowing the growth and the expansion of the tumor. In particular, TGF- $\beta$ promotes the differentiation of the Treg positive cells, this increasing their total circulating number. This represents the scientific rationale for combining immunotherapeutic agents, such as immune check points inhibitors, with galunisertib in the treatment of HCC. Currently, a clinical trial combining galunisertib with an anti-PD-1 inhibitor, NCT02423343, is ongoing and the results will soon be available. Nevertheless, it has not yet been demonstrated that TGF- $\beta$ is directly responsible for the recruitment of Treg cells into the surrounding microenvironment, nor has any direct correlation between TGF- $\beta$ concentration and Treg expression been demonstrated.

In conclusion, the prominent protumorigenic effect of TGF- $\beta$ in liver cancer, as well as the promising results using galunisertib in Phase II clinical trials, suggest that the TGF- $\beta$ pathway is a relevant druggable target for patients with advanced HCC. The challenge for the future will be to better define the patient cohort who will benefit from such a therapy.

\section{Financial \& competing interests disclosure}

The IT-LIVER consortium was created thanks to a grant from the People Programme (Marie Curie Actions) of the FP7-2013, under REA grant agreement \#PITN-GA-2012-316549. The authors have no other relevant affiliations or financial involvement with any organization or entity with a financial interest in or financial conflict with the subject matter or materials discussed in the manuscript apart from those disclosed.

No writing assistance was utilized in the production of this manuscript.

\section{References}

1 Fabregat I, Moreno-Cáceres J, Sánchez A et al. TGF- $\beta$ signaling and liver disease. FEBS J. 283, 22119-2232 (2016).

2 Dewidar D, Soukupova J, Fabregat I et al. TGF- $\beta$ in hepatic stellate cell activation and liver fibrogenesis: updated. Curr. Pathobiol. Rep. 3, 291-305 (2015).

3 Crosas-Molist E, Fabregat I. Role of NADPH oxidases in the redox biology of liver fibrosis. Redox Biol. 6, 106-111 (2015).

4 Fischer AN, Fuchs E, Mikula M et al. PDGF essentially links TGF-beta signaling to nuclear beta-catenin accumulation in hepatocellular carcinoma progression. Oncogene 26, 3395-3405 (2007).

5 Caja L, Sancho P, Bertran E et al. Dissecting the effect of targeting the epidermal growth factor receptor on TGF-beta-induced apoptosis in human hepatocellular carcinoma cells. J. Hepatol. 55, 351-358 (2011).

6 Giannelli G, Rani B, Dituri F et al. Moving towards personalized therapy in patients with hepatocellular carcinoma: the role of the microenvironment. Gut 63, 1668-1676 (2014).
7 Giannelli G, Koudelkova P, Dituri F et al. Role of epithelial to mesenchymal transition in hepatocellular carcinoma. J. Hepatol. 65, 798-808 (2016).

8 Malfettone A, Soukupova J, Bertran E et al. Transforming growth factor- $\beta$-induced plasticity causes a migratory stemness phenotype in hepatocellular carcinoma. Cancer Lett. 392, 39-50 (2017).

9 Morris SM, Baek JY, Koszarek A et al. Transforming growth factor-beta signaling promotes hepatocarcinogenesis induced by p53 loss. Hepatology 55, 121-131 (2012).

$10 \mathrm{Mu} X$, Pradere JP, Affò $S$ et al. Epithelial transforming growth factor- $\beta$ signaling does not contribute to liver fibrosis but protects mice from cholangiocarcinoma. Gastroenterology 150, 720-733 (2016).

11 Coulouarn C, Factor VM, Thorgeirsson SS. Transforming growth factor-beta gene expression signature in mouse hepatocytes predicts clinical outcome in human cancer. Hepatology 47, 2059-2067 (2008).

12 Fransvea E, Angelotti U, Antonaci S et al. Blocking transforming growth factor-beta upregulates E-cadherin and reduces migration and invasion in hepatocellular carcinoma cells. Hepatology 47, 1557-1566 (2008).
13 Bertran E, Crosas-Molist E, Sancho P et al. Overactivation of the TGF-beta pathway confers a mesenchymal-like phenotype en CXCR4-dependent migratory properties to liver tumor cells. Hepatology 58, 2032-2044 (2013).

14 Hoshica Y, Nijman SM, Kobayashi M et al. Integrative transcriptome analysis reveals common molecular subclasses of human hepatocellular carcinoma. Cancer Res. 69, 7385-7392 (2009).

15 Giannelli G, Mikulits W, Dooley S et al. The rationale for targeting TGF- $\beta$ in chronic liver diseases. Eur. J. Clin. Invest. 46, 349-361 (2016).

16 Maier A, Peille AL, Vuaroqueaux V et al. Anti-tumor activity of the TGF-beta receptor kinase inhibitor galunisertib (LY2157299 monohydrate) in patient-derived tumor xenografts. Cell Oncol. (Dordr.) 38, 131-144 (2015).

17 Fransvea E, Mazzocca A, Santamato A et al. Kinase activation profile associated with TGF-beta-dependent migration of HCC cells: a preclinical study. Cancer Chemother. Pharmacol. 68, 79-86 (2011).

18 Giannelli G, Villa E, Lahn M. Transforming growth factor-beta as a therapeutic target in 
EDITORIAL Fabregat \& Giannelli

hepatocellular carcinoma. Cancer Res. 74 1890-1894 (2014).

19 Dituri F, Serio G, Filannino D et al. Circulating TGF-beta1-related

biomarkers in patients with hepatocellular carcinoma and their association with HCC staging scores. Cancer Lett. 353, 264-271 (2014).

20 Cao Y, Agarwal R, Dituri F et al. NGS-based transcriptome profiling biomarkers for companion diagnostic of the TGF-b receptor blocker galunisertib in HCC. Cell Death Dis. 8, e2634 (2017). 